\title{
D-branes and coherent topological charge structure in QCD
}

\section{H. B. Thacker*}

Physics Department, University of Virginia, Charlottesville, VA 22904, USA

E-mail: hbt8r@virginia.edu

Monte Carlo studies of pure glue $S U$ (3) gauge theory using the overlap-based topological charge operator have revealed a laminar structure in the QCD vacuum consisting of extended, thin, coherent, locally 3-dimensional sheets of topological charge embedded in 4D space, with opposite sign sheets interleaved. Studies of localization properties of Dirac eigenmodes have also shown evidence for the delocalization of low-lying modes on effectively 3-dimensional surfaces. In this talk, I review some theoretical ideas which suggest the possibility of 3-dimensionally coherent topological charge structure in 4-dimensional gauge theory and provide a possible interpretation of the observed structure. I begin with Luscher's "Wilson bag" integral over the 3-index ChernSimons tensor. The analogy with a Wilson loop as a charged world line in 2-dimensional $C P^{N-1}$ sigma models suggests that the Wilson bag surface represents the world volume of a physical membrane. The large-N chiral Lagrangian arguments of Witten also indicate the existence of multiple "k-vacuum" states with discontinuous transitions between k-vacua at $\theta=$ odd multiples of $\pi$. The domain walls between these vacua have the properties of a Wilson bag surface. Finally, I review the AdS/CFT duality view of $\theta$ dependence in QCD. The dual realtionship between topological charge in gauge theory and Ramond-Ramond charge in type IIA string theory suggests that the coherent topological charge sheets observed on the lattice are the holographic image of wrapped D6 branes.

XXIVth International Symposium on Lattice Field Theory

July 23-28, 2006

Tucson, Arizona, USA

\footnotetext{
${ }^{*}$ Speaker.
} 


\section{Introduction}

One of the most important byproducts of the chiral lattice fermion revolution of the late 1990's was a new definition of gauge field topological charge density $q(x)=\left(g^{2} / 16 \pi^{2}\right) \operatorname{TrF} \tilde{F}$ on the lattice. This definition is constructed from an exactly chiral Dirac operator $D$ satisfying GinspargWilson relations. The local pseudoscalar operator given by [1]

$$
q_{o}(x)=\frac{1}{2} \operatorname{Tr} \gamma_{5} D(x, x)
$$

(here the trace is over both color and spin) reduces to $q(x)$ in the continuum limit, and is in many respects a superior definition of topological charge density compared to any ultralocal operator constructed directly from gauge links. Recent studies of topological charge using the overlapbased [2] topological charge density have revealed a type of long-range structure that is profoundly different from what might have been expected in an instanton-based model of the QCD vacuum. The first such study [3] produced the surprising result that the $q(x)$ distribution in a typical Monte Carlo gauge configuration is dominated by extended, coherent, thin 3-dimensional sheets of topological charge. In each configuration, sheets of opposite sign are juxtaposed and are everywhere close together in what can roughly be described as a dipole layer which is spread throughout the 4-dimensional Euclidean space (with various folds and wrinkles). The vacuum is thus permeated with what is locally a laminar structure consisting of alternating sign sheets or membranes of topological charge. The thickness of these membranes is typically a few lattice spacings, independent of the physical mass scale, and thus the membranes apparently become infinitely thin in the continuum limit. This kind of "subdimensional" ordering, where coherence takes place on manifolds of lower dimensionality than spacetime itself, is closely related to the appearance of contact terms in the two-point topological charge correlator. In the continuum, the correlator $G(x)=\langle q(x) q(0)\rangle$ is required by spectral considerations to be negative for any nonzero separation $|x|>0$. In practice (i.e. in Monte Carlo calculations), this requirement places severe restrictions on what type of topological charge fluctuations can be dominant. For example, the negativity of the correlator rules out the dominance of bulk-coherent lumps of topological charge (e.g. finite size instantons), since this would lead to a positive correlator over distances smaller than the instanton size. Since the topological susceptibility can be obtained by integrating the 2-point correlator over all $x$, a positive susceptibility can only arise from a delta-function contact term at the origin. The observed arrangement of thin, nearby layers of $q(x)$ with opposite sign builds up a positive contact term at $x=0$ while maintaining the required negativity of the correlator for finite separation. In recent overlap-based studies, the only models that have been found to be dominated by instantons are the $C P^{1}$ and $C P^{2}$ sigma models [4]. But those models are dominated by small instantons with a radius of order lattice spacing, which goes to zero in the continuum limit. Because of this, the instantons contribute to the positive delta-function contact term, but do not contribute at all to the finite $x$ correlator.

The presence of coherent sheets of topological charge is responsible for the positive contact term in the correlator at $x=0$. The range of this contact term on the lattice is associated with the thickness of the sheets, both being a few lattice spacings and approaching zero in physical units. Fig. 1 shows the topological charge correlator for pure glue SU(3) gauge theory at several values of lattice spacing [5]. In addition to the large positive contact term appearing for $r \equiv|x| \leq 2$, the 


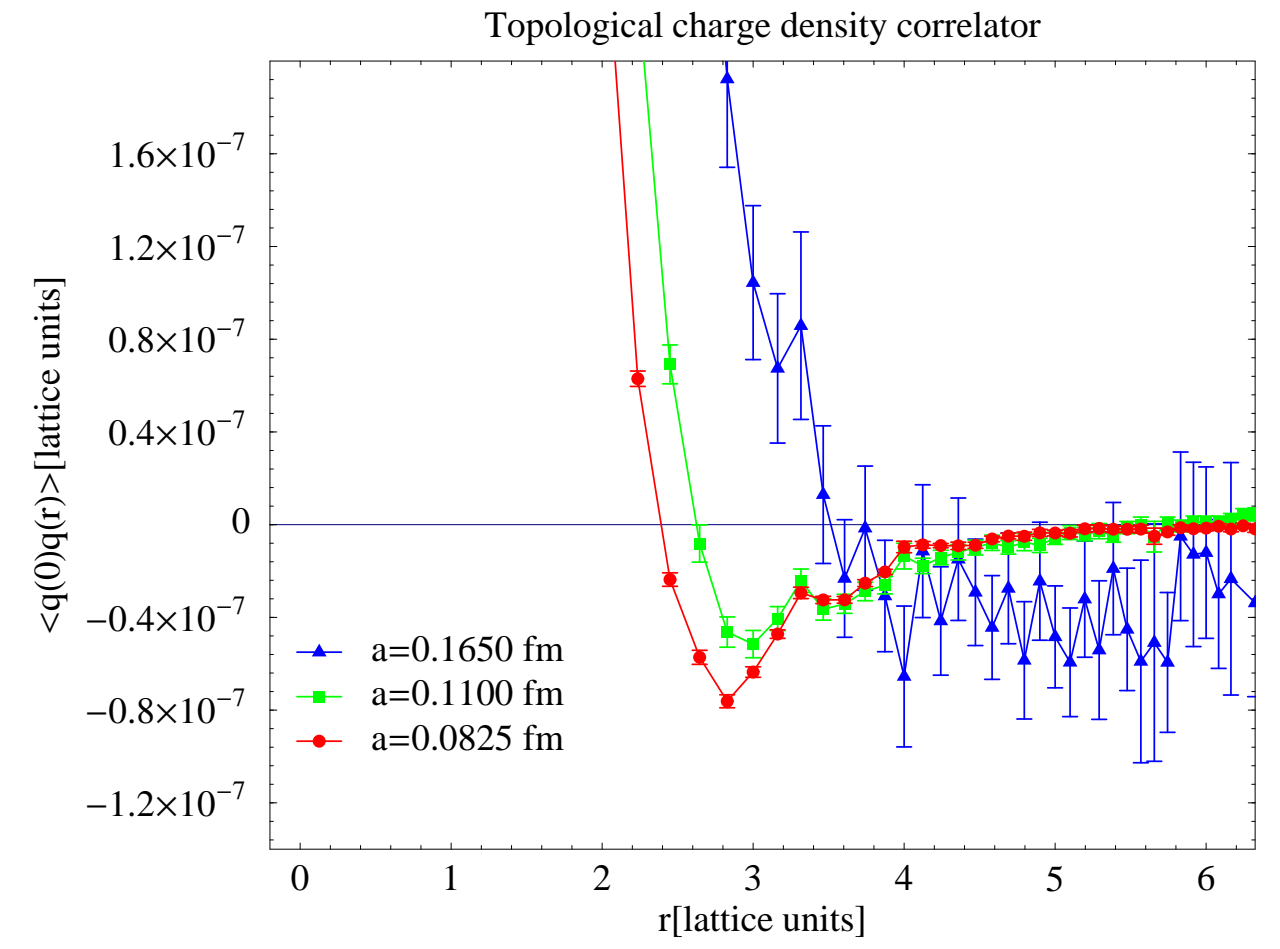

Figure 1: 2-point functions of the overlap based topological charge correlator in pure glue QCD.

other prominent feature is a clear negative dip at a few sites separation. At a calculational level, this negative dip arises from the nearby juxtaposition of positive and negative topological charge layers.

Recent calculations have provided further evidence for the existence of these extended 3dimensional sheets of topological charge [6]. The study of localization properties of low Dirac eigenmodes in Monte Carlo configurations has also shown that low-lying modes are delocalized along effectively 3-dimensional surfaces in 4D space.[7] This is just what one would expect for low Dirac eigenmodes in the presence of 3-dimensional coherent topological charge sheets. Long ago, Diakonov and Petrov [8] pointed out that topological charge fluctuations could lead to a chiral condensate. In the context of the instanton liquid model, the finite density of near-zero eigenmodes required to produce a condensate is generated by approximate 'tHooft zero modes associated with the instantons. But, more generally, coherent regions of positive (negative) topological charge will attract left (right) chiral Dirac modes, and produce low-lying states in which the Dirac mode is bound to the topological charge fluctuation. A rather natural picture for Goldstone boson propagation emerges, in which quarks in a pion "skate" along the surface of a coherent topological charge membrane via Dirac modes which are delocalized along this surface. Further studies of low Dirac eigenmodes and their relationship to the topological charge distribution should help to clarify this picture.

Since the initial discovery of coherent topological charge sheets in QCD, similar methods have been applied to the study of 2-dimensional $C P^{N-1}$ sigma models [9, 4]. For $N>3$, the topological 


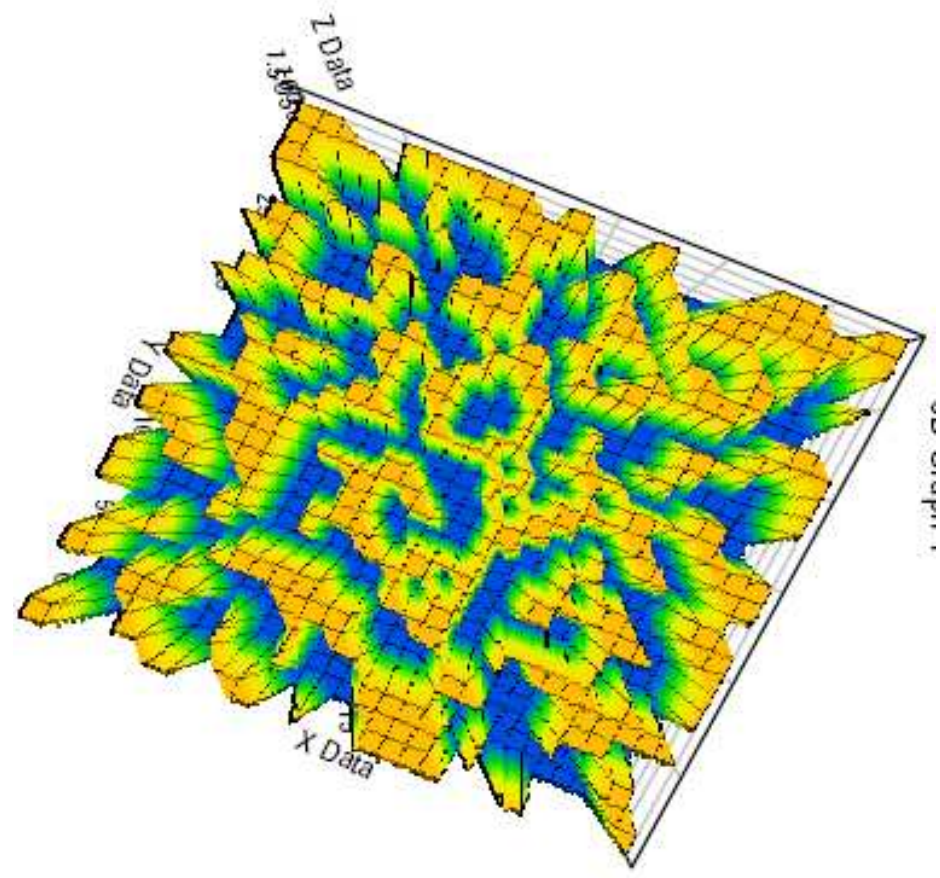

Figure 2: Positive and negative regions of topological charge for a typical $C P^{3}$ configuration.

charge distribution was found to be dominated by thin 1-dimensionally coherent membrane-like structures with interleaved membranes of opposite sign. Fig. 2 shows a typical overlap-based topological charge distribution for $C P^{3}$, where $\operatorname{sign}(q(x))$ is plotted. As I will discuss, this interleaved arrangement of approximately 1-dimensional coherent regions is exactly what one would expect as the analog of the 3-dimensional structures in 4D gauge theory. In both cases, the coherent structure has codimension 1, i.e. the dimensionality of a domain wall.

\section{Wilson loops, Wilson bags, and Chern-Simons tensors}

Though there is now considerable numerical evidence for the existence of 3-dimensionally coherent structure in 4D gauge configurations, the theoretical significance of this structure is far from clear. In this talk I will review a number of related theoretical developments which lead to a plausible interpretation of the observed structure. In fact, I will argue that the Monte Carlo observations essentially support and clarify a view of topological charge in QCD which was suggested long ago by work of Luscher [10] and Witten $[11,12]$ and has re-emerged more recently in the context of AdS/CFT string/gauge duality.

Luscher's discussion begins with the fact that nonzero topological susceptibility implies the presence of a massless pole in the two-point correlator of the Chern-Simons current. Let us define the abelian 3-index Chern-Simons tensor

$$
A_{\mu v \rho}=-\operatorname{Tr}\left(B_{\mu} B_{v} B_{\rho}+\frac{3}{2} B_{[\mu} \partial_{v} B_{\rho]}\right)
$$


where $B_{\mu}$ is the Yang-Mills gauge potential. We consider the Chern-Simons current that is dual to this tensor,

$$
j_{\mu}^{C S}=\frac{1}{32 \pi^{2}} \varepsilon_{\mu v \rho \sigma} A_{v \rho \sigma} .
$$

Although $j_{\mu}^{C S}$ is not gauge invariant, its divergence is the gauge invariant topological charge density

$$
\partial_{\mu} j_{\mu}^{C S}=\frac{1}{16 \pi^{2}} \operatorname{Tr} F \tilde{F}=q(x) .
$$

Choosing a covariant gauge, $\partial_{\mu} A_{\mu v \rho}=0$, the correlator of two Chern-Simons currents has the form

$$
\left\langle j_{\mu}^{C S}(x) j_{v}^{C S}(0)\right\rangle=\int \frac{d^{4} q}{(2 \pi)^{4}} e^{-i q \cdot x} \frac{q_{\mu} q_{v}}{q^{2}} G\left(q^{2}\right) .
$$

From (2.3) we see that $G\left(q^{2}\right)$ must have a $q^{2}=0$ pole whose residue is the topological susceptibility,

$$
G\left(q^{2}\right) \sim \frac{\chi_{t}}{q^{2}}
$$

This long-range correlation constitutes a "secret long-range order" of gauge fields associated with their topological charge fluctuations. Since the CS current is not gauge invariant, the presence of a $q^{2}=0$ pole does not imply the existence of a massless particle (and pure-glue QCD certainly doesn't have one). On the other hand, the pole has a gauge invariant residue $\left(\propto \chi_{t}\right)$ and cannot be transformed away. So it characterizes a physically significant long range coherence in the gauge field associated with topological charge fluctuations.

Luscher's analysis of QCD topological structure in terms of Wilson bags can be understood as a generalization of the analysis of similar properties in the 2-dimensional $C P^{N-1}$ sigma models. These models provide a quite detailed 2D analog of the coherent structure observe in 4D QCD. The $C P^{N-1}$ models have a $U(1)$ gauge invariance and have classical instanton solutions which come in all sizes. (Just like pure-glue QCD these models are classically scale invariant and acquire a mass scale via a conformal anomaly.) In the continuum, the Euclidean action is

$$
\mathscr{L}=\left(D_{\mu} z_{i}\right)^{*}\left(D_{\mu} z_{i}\right)
$$

where $D_{\mu}=\partial_{\mu}+i A_{\mu}$, and $z_{i}, i=1, \ldots, N$ is an $N$-component complex vector constrained to $z_{i}^{*} z_{i}=$ 1. In $2 \mathrm{D} \mathrm{U}(1)$ gauge theories like $C P^{N-1}$, the topological charge density in the continuum is just $(1 / 2 \pi) \varepsilon_{\mu v} \partial_{\mu} A_{v}$ and the Chern-Simons current is just the dual of the gauge potential,

$$
j_{\mu}^{C S}=\frac{1}{2 \pi} \varepsilon_{\mu v} A_{v}
$$

Just as in 4D QCD, nonzero topological susceptibility implies the presence of a $q^{2}=0$ pole in the CS current correlator. But in the two-dimensional case, this same pole appears in the $A_{\mu}$ correlator and is responsible for confinement of $U(1)$ charge via a linear coulomb potential. In the $C P^{N-1}$ models, the gauge field $A_{\mu}$ is an auxiliary field which has no kinetic term in the action. It's equation of motion sets it equal to the $U(1)$ current of the matter fields,

$$
A_{\mu}=i\left(z_{i}^{*} \partial_{\mu} z_{i}-\left(\partial_{\mu} z_{i}\right)^{*} z_{i}\right)
$$


However, as can be shown explicitly in the large-N approximation, the quantum effect of closed $z$-loops produces a dynamically generated kinetic term $\propto F_{\mu \nu}^{2}$ in the low-energy effective action of the gauge field. This produces a $q^{2}=0$ pole in the gauge field correlator which gives rise to a linear, confining coulomb potential between test charges, an area law for fractionally charged Wilson loops, and nonzero topological susceptibility.

Thus in 2-dimensional $U(1)$ gauge theories, topological susceptibility and confinement of $U(1)$ charge are equivalent phenomena. An instructive way to illustrate this is to introduce a nonzero $\theta$ term in the action over a two-volume $V$ enclosed by a boundary $C=\partial V$ with $\theta=0$ outside the boundary. After integration by parts, the theta term is equivalent to a Wilson loop around the boundary carrying a charge $\theta / 2 \pi$ :

$$
\exp \left[\frac{i}{2 \pi} \int d^{2} x \theta(x) \varepsilon_{\mu v} F^{\mu v}\right]=\exp \left[\frac{i \theta}{2 \pi} \oint_{C} A \cdot d x\right]
$$

If the topological susceptibility is nonzero

$$
\chi_{t}=\left.\frac{\partial^{2} E(\theta)}{\partial \theta^{2}}\right|_{\theta=0}>0
$$

then for small nonzero $\theta$, the vacuum energy density $E(\theta)$ inside the loop will be greater than that outside the loop, so the Wilson loop will obey an area law,

$$
\langle W(C)\rangle \propto \exp [-(E(\theta)-E(0)) V]
$$

In a Hamiltonian framework, the Wilson loop around the boundary corresponds to applying a background electric field $\theta$ by putting opposite charges at either end of the 1-dimensional spatial box. The topological susceptibility is just the vacuum polarizability with respect to this field. This is essentially Coleman's original interpretation of $\theta$-dependence in the massive Schwinger model [13]. This general picture carries over to the $C P^{N-1}$ models (except for $C P^{1}$ and $C P^{2}$ [4]). The physics is somewhat different in the $C P^{N-1}$ models than it is in the massive Schwinger model because of the fact that $A_{\mu}$ is an auxiliary field without a kinetic term. The confining electric flux tube in the $C P^{N-1}$ case actually represents the polarization of $z$ pairs in the vacuum between the test charges.

On general principles, we expect the energy density $E(\theta)$ of the true vacuum to be periodic in $\theta \rightarrow \theta+2 \pi$. However, this periodicity can arise in two very distinct ways: analytically or nonanalytically. Dilute instanton calculations produce an $E(\theta)$ which is smooth and periodic (polynomial in $\cos \theta$ ). On the other hand, the mechanism described by Coleman in the massive Schwinger model for periodicity as a function of $\theta$ involves a discontinuous "string breaking" at $\theta=\pi$. For $\theta=\pi$, the original vacuum, with $\frac{1}{2}$ a unit of background flux to the right, becomes degenerate with the one with $\frac{1}{2}$ unit of flux to the left. For $\theta>\pi$ it becomes energetically favorable to pop a charged pair out of the vacuum and screen off one unit of flux. The true ground state shifts to the "k-vacuum" with $k=-1$ (where the local value of $\theta$ differs from the applied field by minus one unit of flux.). In two dimensions, a charged particle world line can be regarded as a domain wall between two different vacuum states. As $\theta$ goes from $\pi$ to $2 \pi$, the vacuum energy inside the bag decreases. At $\theta=2 \pi$, the energy inside and outside are equal, the area law vanishes, and the Wilson loop around $C$ is completely screened by the polarization of the vacuum inside $C$. By Eq. 
(2.8) the screened unit-charged Wilson loop around $C$ is a filamentary thread of current, similar to an edge current in a 2-dimensional superconductor. (In Lorentz gauge, $\partial_{\mu} A_{\mu}=0$, this current is conserved.) Since the $q(x)$ distribution is just the curl of the $A_{\mu}$ field, the topological charge distribution associated with a Wilson line excitation is a dipole layer consisting of two opposite sign layers of one-dimensionally coherent topological charge membranes on either side of the Wilson line. This is just the type of structure that is seen in the Monte Carlo configurations.

In specifying the analogy between $2 \mathrm{D} \mathrm{U}(1)$ theories and 4D SU(N) gauge theories, we take the Chern-Simons currents (2.2) and (2.7) to be directly analogous. This means that the gauge field $A_{\mu}$ in the 2D theory should be identified not with the 4-dimensional gauge field, but with the abelian 3-index Chern-Simons tensor (2.1). Like the gauge field $A_{\mu}$ in 2 dimensions, this is dual to the Chern-Simons current (2.2). Similarly, the Wilson loop or line excitations in the 2-dimensional $U(1)$ models correspond not to Wilson loops in $4 \mathrm{D}$, but to "Wilson bags," i.e. integrals of the Chern-Simons tensor over a 3-surface $\Sigma$.

$$
B(\Sigma)=\exp \left[i(\theta / 2 \pi) \int_{\Sigma} A_{\mu v \lambda} d x_{\mu} d x_{\nu} d x_{\lambda}\right]
$$

This is the analog of a Wilson loop in $2 \mathrm{D} U(1)$ in the sense that, if $\Sigma$ is a closed 3-surface that forms the boundary of a 4-dimensional volume $V$, inserting the Wilson bag factor (2.12) in the gauge field path integral is equivalent to including a $\theta$-term in the gauge action over the 4-volume $V$. The discussion of what happens as we vary $\theta$ from 0 to $2 \pi$ is also completely analogous to the screening of the 2D Wilson loop. For a fractional bag charge $\theta / 2 \pi$, with $0<\theta<2 \pi$, the vacuum inside the bag will have a higher energy than the $\theta=0$ vacuum outside. The expectation of the Wilson bag integral thus satisfies a 4-volume law analogous to the area law for the Wilson loop in 2D,

$$
\langle B(\Sigma)\rangle \sim \exp [-(E(\theta)-E(0)) V]
$$

There will thus be "bag confinement," a confining force between the walls of a fractionally charged bag. At $\theta=\pi$, the vacuum inside the bag will undergo a nonanalytic shift corresponding to the transition between two adjacent $k$-vacua, with parameters $\theta$ and $\theta-2 \pi$ respectively. Finally, as we increase $\theta$ from $\pi$ to $2 \pi$, the force between the bag walls decreases. For a unit-charged bag, $\theta=2 \pi$ inside the bag, and the confining force between bag walls disappears. The topological charge is the curl of the Chern-Simons tensor, so for a uniform $A_{\mu \nu \lambda}$ which is nonzero on a flat bag surface, the topological charge distribution is a dipole layer consisting of thin, coherent positive and negative 3-dimensional layers on either side of the bag surface. Like the Wilson line excitations in the $C P^{N-1}$ models, screened unit-charged Wilson bags provide a reasonable model for interpreting the topological charge structure observed in lattice configurations.

\section{Large $\mathbf{N}$ chiral Lagrangians}

As first emphasized by Witten [11], considerations of large $N$ chiral symmetry also point to a picture of the vacuum in $S U(N)$ gauge theories consisting of discrete quasi-vacua separated by domain walls. For large $N$ the size of the chiral $U(1)$ anomaly shrinks like $1 / N$, which justifies treating the flavor singlet $\eta^{\prime}$ meson as a would-be Goldstone boson and including it in the chiral 
Lagrangian. The effect of the anomaly is incorporated in the chiral Lagrangian in the form of an $\eta^{\prime}$ mass term constructed from the $U(1)$ phase of the chiral field,

$$
\mathscr{L}_{\text {anom }}=\frac{\text { const. }}{N}(-i \ln \operatorname{Det} U)^{2}
$$

where $U$ is the $U(3) \times U(3)$ chiral field. The $\eta^{\prime}$ mass term arising from the anomaly has a different structure than a meson mass term coming from explicit chiral symmetry breaking by quark masses, which has the form

$$
\mathscr{L}_{q m} \propto \operatorname{Tr}\left(\chi^{\dagger} U+h . c\right)
$$

where $\chi$ is the quark mass matrix. In terms of the $U(1)$ phase,

$$
\text { Det } U=e^{i \eta}
$$

the anomaly term (3.1) is a purely quadratic mass term $\propto \eta^{2}$, unlike the quark mass term (3.2) which includes higher order multi-pion interactions and is a single valued function of $U$. The form (3.1) for the anomaly term is dictated by large-N arguments and/or OZI phenomenology, in which multiple-hairpin vertices are suppressed.

In fact, it is the multivaluedness of the logarithm in (3.1) which leads to the appearance of multple k-vacua and domain walls. To illustrate the point in it's simplest form, consider the case of 1-flavor QCD, where the chiral field $U$ reduces to a single $U(1)$ phase, the would-be Goldstone field,

$$
U \rightarrow e^{i \eta}
$$

Now we consider the effective potential for the phase field $\eta$, including both the quark mass term and the anomaly term. The potential is of the form

$$
\mathscr{V}=\mathscr{V}_{0}(\cos \eta)+\frac{m_{0}^{2}}{N} \eta^{2}
$$

where $m_{0}^{2}$ is of order $\Lambda_{Q C D}$. We assume that the potential term $\mathscr{V}_{0}$ has a minimum at $\eta=0$ and is periodic in $\eta \rightarrow \eta+2 \pi$. In the large $\mathrm{N}$ limit the anomaly term can be treated as small, and the potential $\mathscr{V}$ has many nearly-degenerate minima, where the field $\eta$ differs by integer multiples of $2 \pi$. But the chiral anomaly allows us to equate a chiral $U(1)$ phase rotation with a shift of the $\theta$ parameter. This leads to the conclusion that the quasi-vacua identified from the potential (3.5) are $\mathrm{k}$-vacua with $\theta$ parameters differing by integer multiples of $2 \pi$. Thus large- $\mathrm{N}$ chiral Lagrangian considerations lead us to a picture of $\theta$-dependence with Wilson bags separating multiple, nearly degenerate k-vacuum states characterized by effective local values of $\theta$ which differ by integer multiples of $2 \pi$. Although a chiral Lagrangian framework was invoked to arrive at this picture, it is reasonable to conclude that the picture applies even to pure-glue QCD without quarks. The quark only serves as a probe of the topological structure of the gauge field via it's chiral phase.

\section{Theta dependence in Yang-Mills theory from string/gauge holography}

A profound new source of intuition into the long rang structure of 4-dimensional gauge theories has emerged over the last decade in the framework of AdS/CFT string/gauge duality. As 
Witten showed [14], the string/gauge correspondence has particularly interesting implications for the structure of topological charge fluctuations in QCD. It nicely confirms the k-vacuum/domain wall scenario arrived at in the earlier work that I discussed in the last two sections. In fact QCD topological charge provides a particularly direct window on the stringy aspects of gauge theory. This is mainly due to the fact that, in the string/gauge correspondence, topological charge in gauge theory is dual to Ramond-Ramond (RR) charge in type IIA string theory. RR charge is of fundamental importance in string theory. It is similar to magnetic charge in electromagnetism, in that it is a solitonic charge which is not carried by ordinary string states. The fundamental discovery by Polchinski [15] that D-branes carry Ramond-Ramond charge ushered in a new era in string theory where D-branes assumed a central role in the theoretical infrastructure. D-branes were originally conceived for reasons associated with T-duality, which suggested considering open strings with their ends attached to subdimensional hyperplanes with Dirichlet boundary conditions. But it was soon realized that the hyperplanes so defined were actual physical objects that carried energy density, were flexible, and could support local oscillations. The low energy world-volume theory which describes the small oscillations of a D-brane is typically a supersymmetric gauge theory. Much of the technology for studying the connections between string theory and gauge theory is based on various "brane constructions" obtained by considering string theory in the presence of one or more branes (usually, but not always, flat, parallel or superimposed, and filling some $(D+1)$-dimensional subspace of 10-dimensional space.). By superimposing $N$ D-branes in the same subspace, we obtain a world volume theory with a $U(N)$ gauge group, where the gauge bosons correspond to short pieces of string connecting pairs of D-branes. With the interpretation of the $N$ of the gauge group as the number of branes, an interesting thing typically happens in the large $\mathrm{N}$ limit. The gravitational mass of the N D-branes becomes large enough to form a black hole in the dimensions transverse to the branes. From studies of string theory near the horizon of a black hole, Maldacena [16] was led to his famous conjecture that 4-dimensional $\mathscr{N}=4$ supersymmetric $S U(N)$ gauge theory is not simply the low energy limit of a string theory, but is in fact "holographically" equivalent to type IIB string theory in the space $A d S_{5} \times S_{5}$. The idea that gauge theory in 4 dimensions is a holographic representation of a higher-dimensional string theory is not only intriguing but has already gone a long way toward illuminating some of the partial understandings of QCD that have been around for many years. In the gravitational context, the idea of holography has its origin in the observation by Beckenstein and Hawking that the entropy of a black hole is proportional not to it's volume but to the surface area of it's horizon. It is as if everything that went on inside the black hole was uniquely encoded on it's horizon. In the AdS/CFT correspondence, the behavior of weakly coupled string theory in the 5-dimensional $A d S$ space maps holographically to a strongly coupled supersymmetric gauge theory on the 4-dimensional boundary of that space.

In the equivalence conjectured by Maldacena, the 4-dimensional gauge theory that is equivalent to IIB string theory in $A d S_{5} \times S_{5}$ is a conformally invariant field theory, $\mathscr{N}=4$ supersymmetric Yang Mills. In Anti-deSitter space, conformal symmetry of the corresponding gauge theory is generic. However, Witten showed that, with an appropriate arrangement of D-branes and boundary conditions, it is possible to establish a similar holographic equivalence between string theory and ordinary, nonsupersymmetric, asymptotically free gauge theory in 4 dimensions. Gravitationally speaking, Witten's construction replaces the pure $A d S$ space of Maldacena with a Schwarzchild black hole metric, (which arises naturally in the large-N brane construction). It is possible to 
view Witten's construction as an AdS/CFT correspondence, but only by going to 11 dimensional M-theory. An equivalent but somewhat more direct path to 4-dimensional QCD is a brane construction $[17,14]$ which begins with a stack of $N$ 4-branes in 10-dimensional IIA string theory on the spacetime manifold $R_{4} \times S_{1} \times R_{5}$. The 4-branes (which have 5 spacetime dimensions) fill the subspace $R_{4} \times S_{1}$, i.e. they are wrapped around the compact dimension, with supersymmetry breaking boundary conditions imposed on the $S_{1}$. In the dimensions transverse to the 4-branes, this induces a 5-dimensional black hole metric, and the global geometry changes from $R_{4} \times S_{1} \times R_{5}$ to $R_{4} \times D \times S_{4}$, where $D$ is a 2-dimensional disk with a Schwarzchild singularity at its center. The $R_{4}$ is interpreted as $4 \mathrm{D}$ spacetime. For our purposes, the $S_{4}$ plays an essentially passive role except as a place to wrap 6-branes. In the discussion of holography and QCD topological charge, the crucial concept provided by Witten's brane construction is the disk $D$ attached to each point in 4-dimensional spacetime. To understand how $\theta$-dependence of QCD arises in the string theory context, we note that the world-volume theory on the 4-branes is actually a 5-dimensional gauge theory on $R_{4} \times S_{1}$. Being odd-dimensional, this world volume theory generically includes a 5-dimensional Chern-Simons term which has the form

$$
\mathscr{L}_{C S_{5}}=\frac{1}{8 \pi^{2}} a \wedge \operatorname{Tr}(F \wedge F)
$$

where $\operatorname{Tr}(F \wedge F)$ is the topological charge density in the 4-dimensional theory, and $a$ is the RamondRamond $U(1)$ field around the compact $S_{1}$. In the limit of small compactification radius, the 5D Chern-Simons term reduces (at least locally) to a 4D theta term

$$
\mathscr{L}_{C S_{5}} \rightarrow \frac{\theta}{16 \pi^{2}} F \wedge F
$$

where the value of $\theta$ is given by the line integral of the RR $U(1)$ field around the compact dimension,

$$
\theta=\oint_{S_{1}} a_{5} d x_{5}
$$

Here $S_{1}$ is around the perimeter of the disk $D$. Thus $\theta$ is proportional to the amount of RamondRamond flux threaded through the singularity at the center of the disk:

$$
\theta=\int_{D} f_{\mu v} d x_{\mu} d x_{v}
$$

where

$$
f_{\mu \nu}=\partial_{\mu} a_{v}-\partial_{\nu} a_{\mu}
$$

In the holographic framework, the radius of the compact $S_{1}$ serves as an ultraviolet cutoff, rather analogous to the lattice spacing in a lattice formulation. In the limit of small radius, the value of $\theta$ defined by the line integral (4.3) will approach a spacetime constant $\theta$ parameter, but only $\bmod 2 \pi$. Different regions of space may be in different $\mathrm{k}$-vacua, i.e. have values of $\theta$ which differ by multiples of $2 \pi$ and thus are separated by domain walls. The string theory interpretation of $\theta$ as the Wilson loop of the RR field around the compact dimension thus leads naturally to the existence of k-vacua. It also points to the correct candidate for the string theory analog of a gauge theory Wilson bag. In IIA string theory, a special role is played by D6-branes and I will now argue that the topological charge membranes seen on the lattice can be interpreted as the 


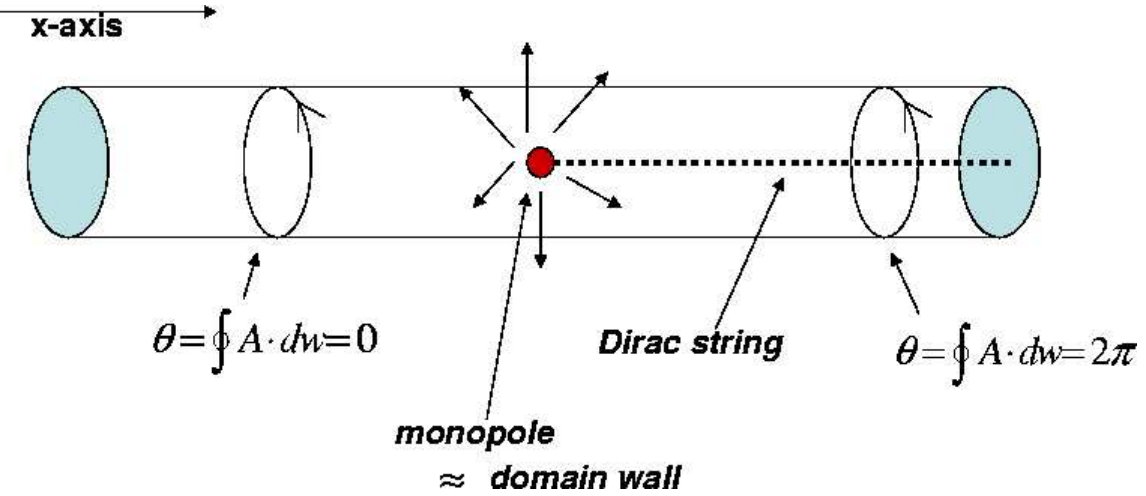

Figure 3: Holographic view of a domain wall between k-vacua in QCD. Plot is at a fixed time, with the long axis of the cylinder representing the spatial coordinate transverse to the domain wall at a fixed time. $w$ is the coordinate on the circle around the compact dimension.

holographic image of D6-branes which are wrapped around the compact $S_{4}$ and therefore appear as 2-branes or membranes in $3+1$ dimensions. I will show that the defining property of the Wilson bag, namely that the value of $\theta$ jumps by $\pm 2 \pi$ when crossing the surface, is in fact nothing but the statement of quantization of Ramond-Ramond charge on a D6-brane. A basic property of the D6-brane is that it carries a quantized amount of RR charge, where this quantization can be demonstrated by a generalization of Dirac's magnetic monopole construction. In general, Dirac's argument involves integrating the magnetic flux over a 2-surface which surrounds or "links with" the object that carries the magnetic charge. Two objects of dimensionality $d_{1}$ and $d_{2}$ which link with each other in $\mathrm{D}$ spatial dimensions satisfy $d_{1}+d_{2}=D-1$. So in $D=9$ spatial dimensions, the spatial dimensionality of an object which can be surrounded by a 2 -surface is $d=6$, i.e. a D6-brane.

A pictorial representation of this situation is shown in Fig. 3. Here the solid cylinder represents the disk $D$ at every point along a spatial axis transverse to the domain wall, (e.g. we put the domain wall in the $y-z$ plane, and the long axis of the cylinder is the $\mathrm{x}$-axis.) This dimensionally reduced picture makes it obvious that the quantized step in $\theta$ across a domain wall comes about in the string theory by a Dirac-type quantization of RR charge. The surface surrounding the 6-brane consists of two disks located on opposite sides of the domain wall (slightly distorted and joined around the outer edge to form a closed surface). The difference between the amount of RR flux going through the two disks, i.e. the step in $\theta$, is quantized.

The picture in Fig. 3 serves equally well to represent a domain wall in the $2 \mathrm{D} C P^{n-1}$ models. At a fixed time, a domain wall is located at a point on the spatial x-axis. Again, the domain wall is analogous to a magnetic monopole with a quantized Dirac string coming out of it.

\section{Small $N$, large $N$, and melting instantons}

As Witten convincingly argued [11], a picture of the QCD vacuum based on instantons is in 
fundamental conflict with expected properties of the large $N$ limit. In fact, in any simple instanton model, the mass of the $\eta^{\prime}$ meson is exponentially suppressed, $\propto \exp (-$ const $\times N)$. But if chiral symmetry breaking occurs as expected in the large $N$ limit, arguments associated with the WittenVeneziano relation imply that the $\eta^{\prime}$ mass $^{2}$ is $\propto 1 / N$. This and other problems with the instanton picture led Witten to suggest that, at sufficiently large $N$, instantons would "melt" due to large field fluctuations associated with the confining vacuum. As we have seen, the idea that topological charge comes in the form of codimension 1 membranes is in a sense the large- $N$ alternative to the instanton picture. The question of whether $N=3$ is large enough for large $N$ arguments to apply to 4-dimensional gauge theory is clearly a central issue. A recent study of $\mathrm{N}$-dependence of topological structure in the $C P^{N-1}$ models [4] provides an interesting perspective on the phenomenon of melting instantons. It turns out that in these models there is a fairly precise instanton melting point $N=N_{c}$ below which instantons dominate the topological charge distribution on the lattice, and topological susceptibility is reasonably well-described by a dilute instanton gas calculation. Above the melting point, instantons disappear and the TC distribution is dominated by coherent codimension 1 surfaces.

For $C P^{N-1}$ with the lattice action used in [4] the instanton melting point is $N_{c} \approx 3.7$. Thus $C P^{1}$ and $C P^{2}$ are below the melting point, and $C P^{3}(N=4)$ is slightly above it. Using the overlapbased $q(x)$, the small instantons which dominate $C P^{1}$ and $C P^{2}$ are quite easily seen, and, at larger values of $\beta$, integer changes of the global charge $Q$ are invariably accompanied by the appearance or disappearance of an identifiable instanton. (Interestingly, the instantons are not visible if the ultralocal plaquette-based definition of $q(x)$ is used.) For reasons first discussed by Luscher [18] the instantons in these models have radii of order lattice spacing. They have zero radius in the continuum limit and result in anomalous scaling of the topological susceptibility. The instanton melting point can be estimated in the dilute gas approximation by measuring the action $\varepsilon$ of a single small instanton on the lattice. Numerically, this is found to be approximately

$$
\varepsilon \approx \frac{N}{2} \times 6.74 \ldots
$$

For $N=1$ and 2 , the instanton contribution falls more slowly than $\mu^{2}$ in the large $\beta$ limit $(\mu=$ mass gap). This results in a divergent topological susceptibility in the continuum limit. On the other hand, if $\varepsilon>4 \pi$ the instanton contribution to $\chi_{t}$ falls off more rapidly than $\mu^{2}$ and becomes negligible compared to scaling contributions which are $\propto \mu^{2}$ Thus there is a sharp transition at $N=N_{c}=8 \pi / 6.74 \approx 3.7$. For $N<N_{c}$, instantons dominate and for $N>N_{c}$ they disappear. The instanton melting point $N_{c} \approx 3.7$, estimated from the dilute gas calculation, agrees very nicely with the direct results of the Monte Carlo calculations, which give a divergent $\chi_{t}$ for $C P^{1}$ and $C P^{2}$ but a $\chi_{t}$ which scales properly for $C P^{3}$ and higher [4].

Using the $C P^{N-1}$ analogy, it is possible to at least crudely estimate the instanton melting point in $4 \mathrm{D} S U(N)$ gauge theory. First we note that the action of a small instanton, and hence the estimated value of $N_{c}$ will change with different lattice actions. So it may be possible to improve the action in a way that would increase the value of $\varepsilon$ and thereby lower the value of $N_{c}$. But Luscher has argued [18] that the lattice effect will always lower the action of a small instanton relative to its continuum value of $\varepsilon_{c}=\frac{N}{2} \times 4 \pi$ So there is a lower bound on the instanton melting point of $N_{c}>2$. (In particular, this means that small instantons cannot be eliminated from $C P^{1}$ 
by improving the action.) The lower-bound estimate of $N_{c}=2$ can be obtained without reference to any particular lattice action. $N_{c}=2$ corresponds to the "tipping point" of the integration over instanton size in a semiclassical instanton calculation. For $N<N_{c}$ the integral diverges at the small instanton end, while for $N>N_{c}$, it diverges for large instantons. For 4-dimensional $S U(N)$ gauge theory, the integral over instanton size behaves (to lowest order in the renormalization group beta function) like

$$
\int \frac{d \rho}{\rho^{5}} \rho^{11 N / 3}
$$

which has it's tipping point at

$$
N_{c}=\frac{12}{11}
$$

Real QCD at $N=3$ is well above this estimate of the instanton melting point, but (5.3) is only a lower bound. For $C P^{N-1}$, the actual melting point (for the simplest action) is nearly twice as large as the lower bound (3.7 vs. 2). In any case, the direct Monte Carlo evidence from $S U(3)$ gauge theory clearly favors the large $N$ scenario of codimension 1 membranes rather than an instanton dominated vacuum.

The phenomenon of melting instantons in the $C P^{N-1}$ models has an amusing interpretation in the framework of string/gauge holography. By analogy with Witten's 4-brane construction, we can imagine that the $\theta$ term in 2D $C P^{N-1}$ arises from a compactified 3-dimensional Chern-Simons term of the form

$$
\mathscr{L}_{C S}=i \varepsilon^{a b c} A_{a} \partial_{b} A_{c}
$$

Here, $a, b, c$ run from 1 to 3 . Let us denote the original spacetime dimensions by 1 and 2 , and the compactified dimension by 3 . Then in the limit of small radius of compactification, the ChernSimons term reduces to a theta term,

$$
\mathscr{L}_{C S} \rightarrow i \frac{\theta}{2 \pi} \varepsilon^{\mu v} \partial_{\mu} A_{v}=i \theta q(x)
$$

where $\mu, v=1,2$, and

$$
\theta=\oint A_{3} d x_{3}
$$

From this 3-dimensional framework, a small instanton in $C P^{1}$ or $C P^{2}$ can be interpreted as a charged particle coupled to the gauge field $A_{3}$ which has a world line which is wrapped around the compact direction in a closed loop, and is pointlike in the 1-2 plane. On the other hand, we may integrate by parts and write,

$$
\mathscr{L}_{C S}=-\frac{i}{2 \pi} \varepsilon_{\mu v}\left(\partial_{\mu} \theta\right) A_{v} \equiv J_{v} A_{v}
$$

where

$$
J_{v} \equiv \frac{1}{2 \pi} \varepsilon_{\mu v} \partial_{\mu} \theta
$$

In this way of writing the CS term, the current $J_{\mu}$ couples to the gauge field in the 1-2 plane. In the limit of small compactification radius, the quantity $\theta$ defined by (5.6) reduces to the constant theta parameter of the 2D theory mod $2 \pi k$. Different k-vacua are separated by domain walls, and the current $J_{\mu}$ is an "edge current" which is nonvanishing along these domain walls.

From this perspective, we could interpret the melting of an instanton as an unwinding of its world line from the compact dimension, with the domain walls of the large $N$ models being the 
remnants of melted or unwound instantons. The basic instability that causes instanton melting is that, for sufficiently large N, they are unstable toward expanding in size. However, they cannot just become large instantons, because that would violate the negativity of the correlator. Instead they expand like a smoke ring and become Wilson line excitations. Most of the time this ring of positive $q$ will be screened by an antiinstanton emerging from the lattice and becoming a concentric ring of negative $q$. forming a dipole layer. This visualization of a melting instanton has the obvious generalization to 4-dimensional QCD, with a small instanton expanding to form a hollow bubble whose surface is a Wilson bag.

\section{6. conclusions}

The Monte Carlo results showing the presence of extended, coherent 3-dimensional topological charge membranes in lattice QCD configurations could have far-reaching implications. Detailed studies of low Dirac eigenmodes and their relation to the topological charge membranes should clarify the role of these membranes in spontaneous chiral symmetry breaking. It is likely that the chiral condensate arises from low eigenmodes associated with these membranes. The chiral anomaly and $\eta$ ' mass insertion suggest a central role of the membranes in inducing quarkantiquark pair creation and annihilation processes in the QCD vacuum. The D-brane picture of the QCD vacuum could provide a better understanding of the OZI rule, in particular the fact that $q \bar{q}$ annihilation within a meson is highly suppressed except in the scalar and pseudoscalar channels. The interpretation of the coherent topological charge sheets as Dbranes also suggests a possible underlying role of supersymmetry in the dynamics of light quarks. Such a connection has already been found in the "supersymmetry relics" discussed recently by Armoni, et al [19]. These predictions are based on a large-N equivalence between $\mathscr{N}=1$ SUSY gauge theory and an orientifold projected theory which, for the case $N=3$ is ordinary non-SUSY 1-flavor QCD. As shown in the poster of Patrick Keith-Hynes at this Conference,[20] the SUSY relic prediction of approximate degeneracy between scalar and pseudoscalar flavor-singlet mesons depends crucially on the mass shifts induced by $q \bar{q}$ annihilation (hairpin) diagrams in the scalar and pseudoscalar channels. The interplay between light quark dynamics and topological charge membranes in QCD is an interesting area for both theoretical and numerical studies. Finally, the interesting question arises whether the D-brane vacuum I have suggested in this talk can explain confinement. This is at least a reasonable possibility, since the presence of topological charge membranes should disorder the vacuum at long distances. A large Wilson loop would have to go through many membranes, which would disorder the color phases around the loop and lead to an area law. Intuition from the string theory side should be useful in addressing these issues.

This work was supported in part by the Department of Energy under grant DE-FG02-97ER41027.

\section{References}

[1] P. Hasenfratz, V. Laliena, F. Niedermayer, Phys. Lett. B427, 125 (1998).

[2] H. Neuberger, Phys. Rev. Lett.81, 4060-4062 (1998).

[3] I. Horvath et al, Phys. Rev. D68, 114505 (2003).

[4] Y. Lian and H. B. Thacker, hep-lat/0607026. 
[5] I. Horváth et al., Phys. Lett. B617: 21 (2005).

[6] E.-M. Ilgenfritz et al., Nucl. Phys. Proc. Suppl. 153: 328 (2006).

[7] MILC Collaboration:C. Aubin et al,, Nucl. Phys. Proc. Suppl. 140: 626 (2005); C. Bernard et al., PoS LAT2005: 299 (2006).

[8] D.I. Diakonov, V.Y. Petrov, Nucl. Phys. B272, 457 (1986).

[9] S. Ahmad, J. T. Lenaghan, H. B. Thacker, Phys. Rev. D72: 114511 (2005).

[10] M. Luscher, Phys. Lett. B78, 465 (1978).

[11] E. Witten, Nucl. Phys. B149, 285 (1979).

[12] E. Witten, Ann. Phys. 128: 363 (1980).

[13] S. Coleman, Ann. Phys. 101: 239 (1976).

[14] E. Witten, Phys. Rev. Lett. 81: 2862 (1998).

[15] J. Polchinski, Phys. Rev. Lett. 75, 4724-4727 (1995).

[16] For a review, see O. Aharony, S. S. Gubser, J. M. Maldacena, H. Ooguri and Y. Oz, Phys. Rept. 323, $183(2000)$.

[17] E. Witten, Adv. Theor. Math. Phys. 2: 505 (1998).

[18] M. Lüscher, Nucl. Phys. B200:61 (1982).

[19] A. Armoni, M. Shifman, and G. Veneziano, Phys. Rev. Lett. 91: 191601 (2003).

[20] P, Keith-Hynes and H. B. Thacker (these proceedings). 